\title{
Hydrogen, boron and nitrogen atoms in diamond: a quantum mechanical vibrational analysis
}

\author{
Francesco Silvio Gentile ${ }^{1} \cdot$ Simone Salustro ${ }^{1} \cdot$ Giulio Di Palma ${ }^{1} \cdot$ Mauro Causà ${ }^{2} \cdot$ Philippe D'Arco $^{3} \cdot$ Roberto Dovesi $^{1}$
}

\begin{abstract}
The structural, electronic and vibrational properties of two common defects in diamond, $\mathrm{CHN}$ and $\mathrm{CHB}$, describing the case in which a carbon $\mathrm{C}_{1}$ atom is substituted by a nitrogen atom, or by a boron atom, breaking a $\mathrm{C}_{1}-\mathrm{C}_{2}$ bond, followed by the saturation of the dangling bond of $\mathrm{C}_{2}$ by a hydrogen atom, are investigated at the quantum mechanical level, by using a periodic supercell approach, hybrid DFT functionals and a local Gaussian-type basis set as implemented in the CRYSTAL code. The effect of concentration of the defects has been explored, by considering two supercells containing 64 and 216 atoms $\left(\mathrm{S}_{64}\right.$ and $\left.\mathrm{S}_{216}\right)$. Formation and hydrogenation energies, geometries, Mulliken charges and the band structure of both defects are reported. The vibrational features of the defects have been investigated, by generating the IR and Raman spectra, and by analyzing graphically and through the isotopic substitution $\left(\mathrm{H} \rightarrow \mathrm{D},{ }^{11} \mathrm{~B} \rightarrow{ }^{10} \mathrm{~B}\right.$ and $\left.{ }^{14} \mathrm{~N} \rightarrow{ }^{15} \mathrm{~N}\right)$ the nature of the most relevant modes related to the defects. The computed $\mathrm{C}-\mathrm{H}$ stretching mode of $\mathrm{CHN}$, once corrected for anharmonicity $\left(3408 \mathrm{~cm}^{-1}\right)$, falls to wavenumbers very close to the experimental peak observed at $3394 \mathrm{~cm}^{-1}$, which can then be reasonably attributed to this specific defect. The present manuscript is included in a special volume in honor and memory of János Ángyán. Although he did not study in particular the kind of defects discussed in the present manuscript, the many methodological contributions he introduced in computational science have inspired many of the tools we have been using here. One of the present authors, $\mathrm{RD}$, in particular, is grateful to János for the illuminating discussions they had in Paris, Nancy and Torino.
\end{abstract}

Electronic supplementary material The online version of this article (https://doi.org/10.1007/s00214-018-2375-0) contains supplementary material, which is available to authorized users.

Francesco Silvio Gentile

francesco.gentile@unina.it

1 Dipartimento di Chimica, Università di Torino and NIS (Nanostructured Interfaces and Surfaces) Centre, Via P. Giuria 5, 10125 Turin, Italy

2 Dipartimento di Ingengeria Chimica, dei Materiali e delle Produzioni Industriali DICMAPI, Università degli Studi di Napoli Federico II, Piazzale Vincenzo Tecchio 80, 80125 Naples, Italy

3 CNRS-INSU, Institut des Sciences de la Terre de Paris, ISTeP UMR 7193, Sorbonne Université, F-75005 Paris, France

\section{Introduction}

Since several decades, diamond has represented a material of high technological relevance, thanks to its extreme physical properties which make it attractive for many applications in different fields, ranging from microelectromechanical systems to heatsinks, laser windows, particle detectors, etc. [1, 2].

Among the large plethora of point defects in diamond, hydrogen is one of the most important impurities. It is included during the growth of natural diamond as well as in chemical vapor deposition (CVD) processes, and it is certainly present at the surfaces. A non-minor fraction, however, is also thought to be incorporated in the bulk. Hydrogen atoms are supposed to be present in several defects [3, 4], involving vacancies and/or isolated or aggregated nitrogen atoms. It is mostly identified through its infrared (IR) active modes (stretching and bending), although probably not all hydrogen atoms present in diamond are IR active.

However, hydrogen is only one of the possible impurities in diamond; among the many, nitrogen and boron are two of the most observed and discussed. 
They can be both present as substitutional defects without leading to substantial distortions to the diamond structure [5].

Single substitutional nitrogen and boron, however, bring a dangling bond on one of the four neighboring carbon atoms, which can be saturated by a hydrogen atom. The resulting defects (CHN and CHB centers) have been largely studied through computational methods and attributed to many experimental features. In particular, the CHN defect has been at first associated [6] with the absorption band at 3107 $\mathrm{cm}^{-1}$, it has recently been firmly established that this mode is the $\mathrm{CH}$ stretching of $\mathrm{VN}_{3} \mathrm{H}$ (a vacancy surrounded by three $\mathrm{N}$ atoms and one $\mathrm{CH}$ group, see Refs. [7, 8]); later on, it has been associated by the same authors [9] to the peak at $3324 \mathrm{~cm}^{-1}$.

The CHB center has been related to the modes at 2664 and $1975 \mathrm{~cm}^{-1}$ through simulations based on the LDA functional [6].

As a result of theoretical developments [10] and computational implementation of fully analytic quantum mechanical methods for the evaluation of infrared and Raman spectra in solids [10-12], computational vibrational spectroscopy is now established as an additional and effective tool in the interpretation of experimental spectra [13-15]. In a series of previous works [7, 16-24] some of the present authors have proposed the quantum-mechanical characterization of several point defects in diamond. In this work, electronic and spectroscopic features of substitutional nitrogen and boron atoms neighboring an interstitial hydrogen are discussed. Calculations are performed within a periodic supercell scheme. The hybrid B3LYP functional, as implemented in the CRYSTAL program [10], is applied. The effects of different defect concentrations are explored.

The paper is structured as follows: Section 2 reports information on the adopted computational setup. Section 3 is organized in two parts. Section 3.1 is devoted to the equilibrium geometry of the studied defects, to their charge distribution and to their band structure. In Sect. 3.2, the vibrational features of the defects are reported and analyzed. In Sect. 4, some conclusions are drawn. Finally, in Supplemental section, the effect of temperature on the hydrogenation process is presented and discussed.

\section{Computational methods}

Calculations have been performed by using the B3LYP global hybrid functional $[25,26]$, as implemented in the CRYSTAL17 program [27]. An all-electron basis set of Gaussian-type functions has been adopted (Pople's 6-21G) for $\mathrm{C}, \mathrm{B}$ and $\mathrm{N}$. The exponent of the most diffuse $s p$ shell of the carbon atom has been optimized in bulk diamond $(0.23$ Bohr $^{-2}$ ); for the other atoms, default values have been used.
For hydrogen, the 6-31G set has been adopted [28]. The insertion energy of the hydrogen atom and the $\mathrm{X}-\mathrm{H}$ anharmonic vibrational frequency have been calculated also with larger basis sets $\left(6-21 \mathrm{G}^{*}, 6-31 \mathrm{G}\right.$ and 6-31G* [29]) and other functionals, ranging from pure (LDA [30] and PBE [31]), to global (PBE0 [32]), and range-separated (HSE06 [33]) hybrids. The truncation of the Coulomb and exchange infinite lattice series is controlled by five thresholds $\mathrm{T}_{i}$ (see CRYSTAL manual, reference [34], for more details), which have been set to $8\left(\mathrm{~T}_{1}-\mathrm{T}_{4}\right)$ and $16\left(\mathrm{~T}_{5}\right)$. The convergence threshold on energy for the self-consistent-field (SCF) procedure has been set to $10^{-8}$ Hartree for structural optimizations and to $10^{-10}$ Hartree for vibration frequency calculations.

The DFT exchange-correlation contribution to the Fock matrix has been evaluated by numerical integration over the unit cell volume. Radial and angular points for the integration grid are generated through Gauss-Legendre radial quadrature and Lebedev two-dimensional angular point distributions. The default pruned grid with 75 radial and 974 angular points has been used, whose accuracy can be measured by comparing the integrated charge density $\left(N_{i}\right)$ in the largest supercell here considered (216 atoms), $N_{i}=1298.028$, with the total number of 1298 electrons in the unit cell.

Supercells of increasing size have been used for simulating the effect of defect concentrations. Two cubic supercells containing, respectively, $64\left(\mathrm{~S}_{64}\right)$ and $216\left(\mathrm{~S}_{216}\right)$ atoms have been considered; for each cell, a different $\Gamma$-centered Pack-Monkhorst grid for sampling the reciprocal space has been used, consisting of $4 \times 4 \times 4=64\left(\mathrm{~S}_{64}\right)$ and $2 \times 2 \times 2=8\left(\mathrm{~S}_{216}\right) \mathbf{k}$-points in the first Brillouin Zone.

\subsection{Harmonic frequencies and the IR and Raman spectra}

Frequencies at the $\Gamma$ point are obtained within the harmonic approximation by diagonalizing the mass-weighted Hessian matrix, $W$, whose elements are defined as [13, 35-38]

$W_{\alpha i, \beta j}^{\Gamma}=\frac{H_{\alpha i, \beta j}^{\mathbf{0}}}{\sqrt{M_{\alpha} M_{\beta}}}$ with $H_{\alpha i, \beta j}^{\mathbf{0}}=\left(\frac{\partial^{2} E}{\partial u_{\alpha i}^{\mathbf{0}} \partial u_{\beta j}^{\mathbf{0}}}\right)$,

where $M_{\alpha}$ and $M_{\beta}$ are the masses of atoms associated with the $i$ and $j$ atomic coordinates. Once the Hessian matrix, $H_{\alpha i, \beta j}^{0}$, has been calculated, frequency shifts due to isotopic substitutions can be calculated readily, at no computational cost, by changing masses in Eq. 1 .

Energy first derivatives with respect to the atomic displacements, $v_{\alpha, j}=\partial E / \partial u_{\alpha, j}$, are calculated analytically for all the $u_{\alpha, j}$ coordinates ( $E$ is the total energy, $u_{\alpha, j}$ is the displacement coordinate with respect to the equilibrium, $\alpha$ labels each atom), whereas second-order derivatives at the equilibrium geometry 
are calculated numerically using a single displacement along each coordinate:

$\left[\frac{\partial v_{\alpha j}}{\partial u_{\beta i}}\right] \approx \frac{v_{\alpha j}\left(0, \ldots, u_{\beta i}, \ldots, 0\right)-v_{\alpha j}(0, \ldots, 0, \ldots, 0)}{u_{\beta i}}$

Previous calculations $[35,39]$ have shown that in bulk systems the influence of $u$ is very small (less than $1 \mathrm{~cm}^{-1}$ ) when $\mathrm{H}$ atoms are not present; much larger effects have been found for katoite [40], brucite [41] and diaspore [42], related to the anharmonicity of the $\mathrm{OH}$ stretching. More generally, anharmonicity is large in all cases in which $\mathrm{H}$ atoms are involved (vide infra).

Integrated intensities for IR absorption $\mathcal{I}_{p}$ are computed for each mode $p$ by means of the mass-weighted effectivemode Born-charge vector $\mathbf{Z}_{p}[43,44]$ evaluated through a CPHF/KS approach: [11, 45]

$\mathcal{I}_{p} \propto\left|\mathbf{Z}_{p}\right|^{2}$

The relative Raman intensities of the peaks are computed analytically by exploiting a scheme illustrated in Refs. [46, 47]. Both schemes are based on the solutions of first- and second-order coupled Perturbed-Hartree-Fock/Kohn-Sham (CPHF/KS) equations [48, 49].

\subsection{The anharmonicity of the $\mathrm{C}-\mathrm{H}$ vibrational frequency}

The importance of anharmonicity in $\mathrm{H}$ containing systems can be appreciated by looking at the anharmonicity constant $\omega_{e} \chi_{e}$ of some simple diatomic molecules such as $\mathrm{H}_{2}, \mathrm{HF}$, $\mathrm{HCl}, \mathrm{HBr}, \mathrm{F}_{2}, \mathrm{Cl}_{2}, \mathrm{Br}_{2}$, where $\omega_{e} \chi_{e}$ is $121,90,53,45,12,3$ and $1 \mathrm{~cm}^{-1}$, respectively [50].

As regards the $\mathrm{C}-\mathrm{H}$ stretching, its value is estimated to be around $60 \mathrm{~cm}^{-1}$ for many organic molecules [51, 52].

Experimentally, when both the fundamental frequency $\omega_{01}$ and the first overtone $\omega_{02}$ are available, $\omega_{e} \chi_{e}$ can be obtained through the formula

$\omega_{e} \chi_{e}=\frac{\left(2 \omega_{01}-\omega_{02}\right)}{2}$

that permits also the definition of the harmonic frequency $\omega_{e}$ as:

$\omega_{e}=\omega_{01}+2 \omega_{e} \chi_{e}$

These formulae are derived from the eigenvalues of the Morse potential $U_{M}(r)=D\left[1-e^{-\alpha\left(r-r_{e}\right)}\right]^{2}$, which are:

$E_{n}=\omega_{e}\left(\frac{n+1}{2}\right)-\omega_{e} \chi_{e}\left(\frac{n+1}{2}\right)^{2}$

and, as usual:

$\omega_{01}=E_{1}-E_{0}$ and $\omega_{02}=E_{2}-E_{0}$
$\mathrm{CH}, \mathrm{NH}$ and in particular $\mathrm{OH}$ bonds show large anharmonic effects. These groups are quite common in molecules and molecular crystals. The last one plays an important role in crystalline solids, as it is present in many different types of compounds, spanning from ionic (like metal hydroxides) to semi-covalent (like silicates and aluminosilicates).

\section{Results}

\subsection{Geometry, charge distribution, band structure and formation energy}

In the top-left panel of Fig. 1, the conventional cell of diamond is shown, in which a $\mathrm{CH} \Sigma$ defect is created, where $\Sigma$ stands for B (Fig. 1a) or N (Fig. 1b). Bond distances (in $\AA$ ), atomic net charges $Q$ (in $|e|$ ) and bond populations (BP, in $|e|)$ are reported. $\mathrm{Q}$ and $\mathrm{BP}$ are obtained from a Mulliken analysis of the charge distribution.

The local symmetry of $\mathrm{CHN}$ is $\mathrm{C}_{3 v}$, the $\mathrm{C}_{3}$ rotation axis being oriented along the $\mathrm{C}_{1}-\mathrm{H}-\mathrm{N}$ line. In $\mathrm{CHB}$, on the contrary, the $\mathrm{H}$ atom is not on the line connecting $\mathrm{C}_{1}$ and $\mathrm{B}$ (the angle is $146^{\circ}$ instead of $180^{\circ}$ ), and occupies a general position. This has important consequences, in particular for the IR and Raman spectra. The $\mathrm{C}_{1}-\mathrm{H}$ distance is $1.038 \AA$ in $\mathrm{CHN}$, and $1.215 \AA$ in $\mathrm{CHB}$, with a bond population that reduces from $0.276|e|$ in the former, to $0.144|e|$ in the latter. Then, in $\mathrm{CHB}$, the $\mathrm{C}_{1} \mathrm{H}$ bond is longer, and less covalent than in $\mathrm{CHN}$. The $\mathrm{N}-\mathrm{H}$ distance is $1.325 \AA$ (steric effect due to the lone pair), whereas the $\mathrm{B}-\mathrm{H}$ distance is much shorter, $1.066 \AA$.

The $\mathrm{C}_{1}-\mathrm{H}$ bond is strongly polarized in $\mathrm{CHN}$, as a consequence of the presence of the $\mathrm{N}$ lone pair, oriented toward the $\mathrm{H}$ atom: the net charges on $\mathrm{H}$ being +0.31 versus +0.23 $(\mathrm{CHB})$, and -0.20 versus $-0.13|e|$ on $\mathrm{C}_{1}$. The $\mathrm{N}$ net charge is $-0.56|e|$, and is to a large amount compensated by the three nearest carbon atoms $(+0.15 \times 3=+0.45|e|)$.

The $\mathrm{B}$ net charge is positive, $+0.48|e|$, mostly compensated by the negatively charged $\mathrm{C}$ first neighbors (overall $-0.45|e|)$. So it turns out that the $\mathrm{H}$ atom in $\mathrm{CHN}$ is attracted by the concentrated electronic cloud of the $\mathrm{N}$ lone pair ( $\mathrm{H}$ is positively charged), whereas in $\mathrm{CHB}$ it is repelled by the positively charged $\mathrm{B}$ atom. In order to reduce this repulsion, it deflects from the $\mathrm{C}_{1} \mathrm{~B}$ line. The $\mathrm{N}-\mathrm{H}$ bond population is very small and negative $(-0.003|e|$, slightly repulsive), whereas the $\mathrm{B}-\mathrm{H}$ one is strongly negative $(-0.190|e|)$

The equilibrium geometries obtained with the $S_{216}$ supercells are very similar to the one shown in Fig. 1, the only exception being the $\mathrm{BHC}_{1}$ angle, which reduces from $146,02^{\circ}$ to $130.77^{\circ}$. This is the reason why the vibrational modes of $\mathrm{CHB}$ are more sensitive than the $\mathrm{CHN}$ ones to the defect concentration. 
Fig. 1 Schematic representation of the $\mathrm{CH} \Sigma$ defect in diamond, where $\Sigma$ stands for boron (a) or nitrogen (b). B3LYP equilibrium distances (large, in $\AA$ ), net charges (large, bold, in $|e|)$ and bond populations (italic, in $|e|$ ), as resulting from a Mulliken analysis, are shown. Data refer to $\mathrm{S}_{64}$

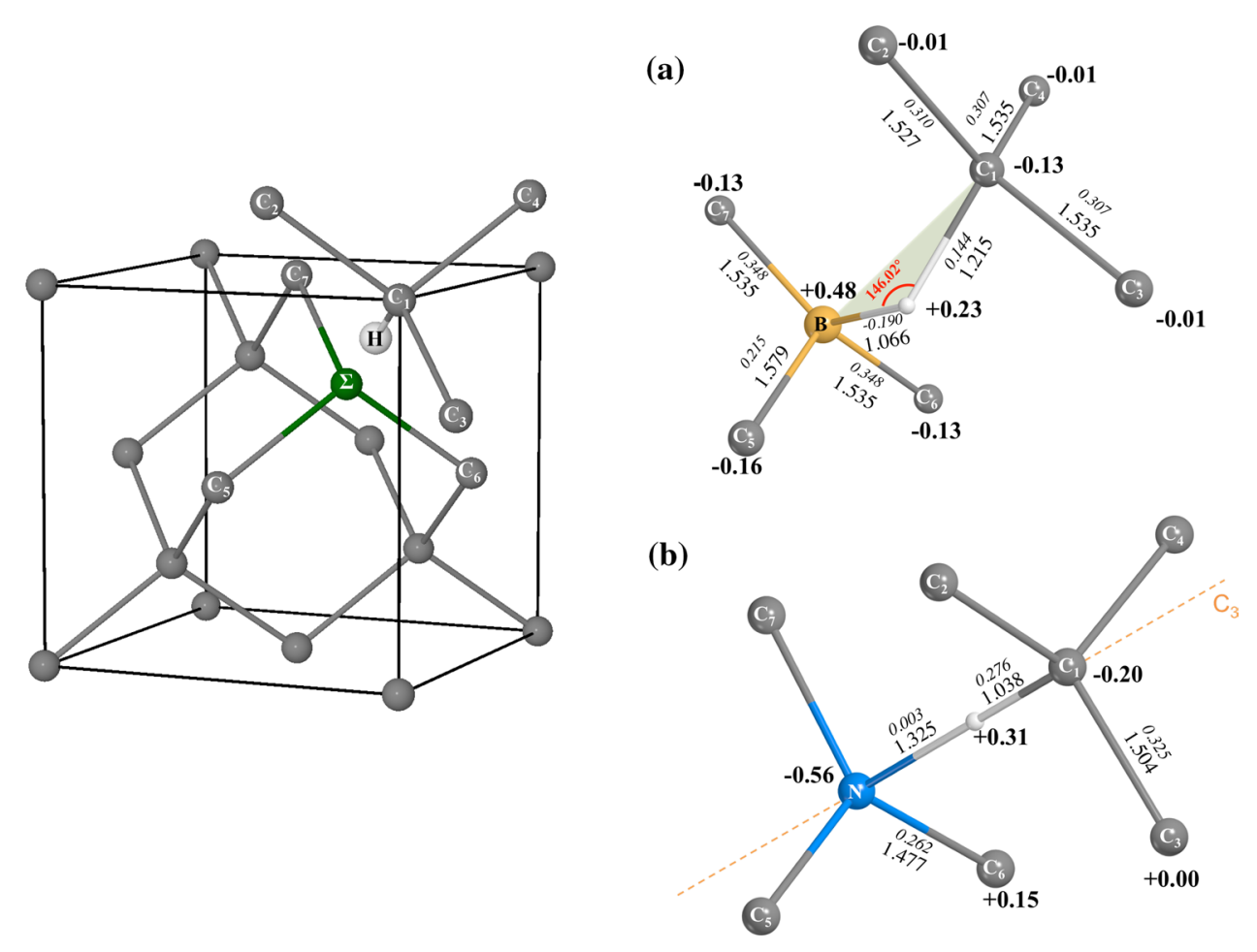

The band structures of the CHN and CHB defects are compared to the one of pristine diamond in Fig. 2, where the most relevant energy differences are shown. The band gap of the latter, $5.75 \mathrm{eV}$ at the B3LYP level, is in good agreement with the experimental value of $5.80 \mathrm{eV}$ [53]. The main difference between the $\mathrm{CHN}$-defective and the perfect diamond is the band that appears in the gap, above the highest occupied band of perfect diamond, and due to the $\mathrm{N}$ lone pairs. The corresponding anti-bonding band is just below the lowest unoccupied diamond band. The calculated indirect band gap of $\mathrm{CHN}$ is $4.9 \mathrm{eV}$.

The CHB defect introduces an empty band just below the diamond conducting band, without any significant modification of the valence bands. The resulting direct band gap is $5.0 \mathrm{eV}$.

Let us consider now the formation energy $\mathrm{E}_{f}$ of the defect. In Eq. 8, a C atom leaves the diamond supercell and two atoms, i.e., one hydrogen and one nitrogen (or boron) atoms, enter in the supercell. According to this reaction, the CHB defect turns out to be more stable than CHN (see Table 1): formation energies obtained with hybrid functionals are around $-4.8 \mathrm{eV}$ for $\mathrm{CHB}$ and $-1.8 \mathrm{eV}$ for CHN. The Hartree-Fock values are higher (less negative) by 2.5 (CHB) and 4.2 (CHN) eV, whereas PBE and LDA are lower by up to 1.5 and $2.2 \mathrm{eV}$.

The hydrogenation process, described by Eq. 9, is exothermic for all functionals here adopted. The insertion of one hydrogen atom is easier in $\mathrm{CHN}$, where hydrogenation energies $\mathrm{E}_{H}$ obtained with the hybrid functionals are around $-1.3 \mathrm{eV}$, than in $\mathrm{CHB}$, where $\mathrm{E}_{H}$ for hybrids are $0.3-0.4 \mathrm{eV}$ higher. The lower energy for $\mathrm{CHN}$ is due to the strong electrostatic attraction between the $\mathrm{N}$ lone pair and the positively charged $\mathrm{H}$ atom.

In order to have reasonable formation energies, the same basis set quality must be adopted in the defective solid and in the isolated atoms. For this reason, the latter have been inserted in a cluster formed by their first, second and third stars of neighbors in the bulk; these atoms have then been transformed in "ghosts." (Electron and nuclei are eliminated, and only the basis functions are kept.) In this way, most of the basis set superposition error (BSSE) is corrected for. Some thermodynamic properties have been evaluated considering the phonons at the $\Gamma$ point only $[54,55]$.

In Supplementary Material section, $\Delta \mathrm{G}$ (Gibbs free energy) for Eq. 9 as a function of temperature is reported in the $100-10,000 \mathrm{~K}$ interval, and the stability of the two defects is evaluated and discussed.

Diamond $+\mathrm{H}+\Sigma \stackrel{\mathrm{E}_{f}}{\rightleftharpoons} \mathrm{CH} \Sigma+\mathrm{C}, \quad(\Sigma=\mathrm{B}, \mathrm{N})$

$\mathrm{C} \Sigma+\mathrm{H} \stackrel{\mathrm{E}_{\mathrm{H}}}{\rightleftharpoons} \mathrm{CH} \Sigma, \quad(\Sigma=\mathrm{B}, \mathrm{N})$ 
Table 1 Defect formation energy $E_{\mathrm{f}}$ (Eq. 8) and $\mathrm{C}-\mathrm{H}$ homolytic cleavage energy $E_{\mathrm{H}}$ (Eq. 9) for the $\mathrm{CHB}$ (top data) and CHN (bottom data) defects in diamond, as computed with different functionals. $\mathrm{E}(\mathrm{H}), \mathrm{E}(\mathrm{B}), \mathrm{E}(\mathrm{N}) \mathrm{E}(\mathrm{C})$ are the atomic energies evaluated by inserting the atom in a fragment of the crystalline solid in which all the first, second and third neighbors have been transformed in ghost atoms, in order to compensate for the basis set superposition error. All values correspond to the $\mathrm{S}_{64}$ supercells

\begin{tabular}{|c|c|c|c|c|c|c|c|}
\hline Method & $\begin{array}{l}\mathrm{E}(\mathrm{H}) \\
\left(E_{\mathrm{h}}\right)\end{array}$ & $\begin{array}{l}\mathrm{E}(\mathrm{B}) \\
\left(E_{\mathrm{h}}\right)\end{array}$ & $\begin{array}{l}\mathrm{E}(\mathrm{C}) \\
\left(E_{\mathrm{h}}\right)\end{array}$ & $\begin{array}{l}\mathrm{E}(\mathrm{CB}) \\
\left(E_{\mathrm{h}}\right)\end{array}$ & $\begin{array}{l}\mathrm{E}(\mathrm{CHB}) \\
\left(E_{\mathrm{h}}\right)\end{array}$ & $\begin{array}{l}E_{f} \\
(e V)\end{array}$ & $\begin{array}{l}E_{H} \\
(e V)\end{array}$ \\
\hline $\mathrm{HF}$ & -0.4982 & -24.5228 & -2421.7608 & -2409.0305 & -2409.0285 & -2.41 & -0.54 \\
\hline PBE0 & -0.4991 & -24.6084 & -2434.8068 & -2422.0530 & -2422.0530 & -4.97 & -0.98 \\
\hline B3LYP & -0.4969 & -24.6360 & -2435.5789 & -2422.8253 & -2422.8253 & -4.61 & -0.95 \\
\hline HSE06 & -0.4993 & -24.6078 & -2434.7622 & -2422.0072 & -2422.0072 & -4.93 & -0.72 \\
\hline PBE & -0.4974 & -24.5999 & -2434.4882 & -2421.7371 & -2421.7371 & -5.18 & -0.39 \\
\hline LDA & -0.4760 & -24.3426 & -2416.1460 & -2403.4476 & -2403.4476 & -6.40 & -0.81 \\
\hline Method & $\begin{array}{l}\mathrm{E}(\mathrm{H}) \\
\left(E_{\mathrm{h}}\right)\end{array}$ & $\begin{array}{l}\mathrm{E}(\mathrm{N}) \\
\left(E_{\mathrm{h}}\right)\end{array}$ & $\begin{array}{l}\mathrm{E}(\mathrm{C}) \\
\left(E_{\mathrm{h}}\right)\end{array}$ & $\begin{array}{l}\mathrm{E}(\mathrm{CN}) \\
\left(E_{\mathrm{h}}\right)\end{array}$ & $\begin{array}{l}\mathrm{E}(\mathrm{CHN}) \\
\left(E_{\mathrm{h}}\right)\end{array}$ & $\begin{array}{l}E_{f} \\
(e V)\end{array}$ & $\begin{array}{l}E_{H} \\
(e V)\end{array}$ \\
\hline $\mathrm{HF}$ & -0.4982 & -54.3424 & -2421.7608 & -2438.6702 & -2438.6702 & 2.48 & -0.34 \\
\hline PBE0 & -0.4991 & -54.4774 & -2434.8068 & -2451.8046 & -2451.8046 & -1.77 & -1.34 \\
\hline B3LYP & -0.4969 & -54.5107 & -2435.5789 & -2452.5960 & -2452.5960 & -1.78 & -1.42 \\
\hline HSE06 & -0.4993 & -54.4768 & -2434.7622 & -2451.7601 & -2451.7601 & -1.77 & -1.32 \\
\hline PBE & -0.4974 & -54.4621 & -2434.4882 & -2451.5007 & -2451.5007 & -2.50 & -1.76 \\
\hline LDA & -0.4760 & -54.0624 & -2416.1460 & -2433.0773 & -2433.0773 & -3.95 & -2.07 \\
\hline
\end{tabular}
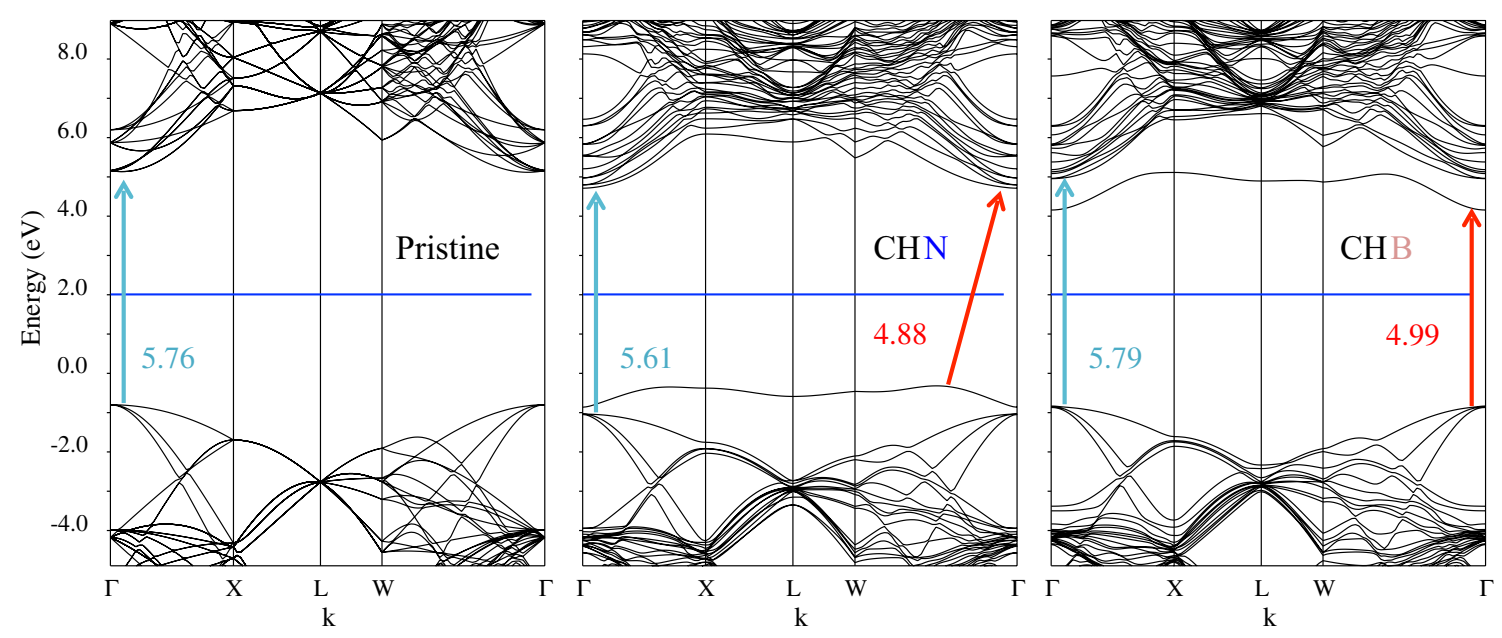

Fig. 2 Band structure of pristine (left) and defective (CHN, central; CHB, right) diamond obtained with the $\mathrm{S}_{64}$ supercell and B3LYP functional. The horizontal blue line indicates the Fermi level. The red arrows represent the optical band gap of the hosting defective level (donor band in $\mathrm{CHN}$ and acceptor band in $\mathrm{CHB}$ ). The turquoise arrows indicate the gap of the hosting diamond. Gaps in $\mathrm{eV}$

\subsection{Infrared and Raman spectra}

Vibrational spectroscopy makes a significant contribution to the identification of the different defects present in diamond. While it is generally rather difficult from the experimental side to assign a given spectral feature unambiguously to a specific structural defect, since, potentially, there is a large number of factors such as defect type, concentration and aggregation, that might hinder or complicate this, the same is not true for a simulation, since the nature of a particular defect, its concentration and local environment i.e., bulk, surface grain boundary and the presence of impurity, are defined precisely a priori.

The Raman spectrum of pristine diamond is simple: it consists of a single and sharp peak at the experimental value of $1332 \mathrm{~cm}^{-1}$; the calculated peak, with the present $6-21 \mathrm{G}$ basis set and B3LYP functional, is at $1317 \mathrm{~cm}^{-1}$. The IR spectrum is even simpler: due to symmetry constraints it does not show any feature. For these reasons, all signals observed in the simulated IR spectrum are thus due to the 
presence of the defect of interest, similarly to those that differ from the one-phonon mode at $1332 \mathrm{~cm}^{-1}$ in the Raman spectrum. Among the many signals that eventually appear, particularly interesting are those that are above the pristine diamond peak value; these are indeed related to vibrational normal modes that involve displacements of just a few set of atoms, typically those of the defect and their nearest neighbors.

\subsubsection{Infrared spectrum}

The IR harmonic vibrational spectra, as computed at two defect concentrations corresponding to the $S_{64}$ and $S_{216}$ supercells, are shown in Fig. 3. Let us comment first the spectra obtained with the largest supercell. In the CHN case, the three hydrogen peaks are well separated from the other peaks: the $\mathrm{A}_{1}$ stretching at $3497 \mathrm{~cm}^{-1}$ and the doubly degenerate bending $E$ mode at $1590 \mathrm{~cm}^{-1}$.

The CHB stretching mode is $3513 \mathrm{~cm}^{-1}$. As a consequence of the $\mathrm{P}_{1}$ symmetry, the two other $\mathrm{H}$ modes in CHB are no more degenerate: one appears at $1866 \mathrm{~cm}^{-1}$ in Fig. 3. What about the third hydrogen mode? Inspection of the graphical animation of the modes, available at the web site indicated at references [56] and [57], clearly shows that a mode involving $\mathrm{H}$ falls down to $364 \mathrm{~cm}^{-1}$. This mode is a sort of nearly free translation of hydrogen, whereas the bending mode at $1866 \mathrm{~cm}^{-1}$, (that is, $276 \mathrm{~cm}^{-1}$ higher than the one of CHN), is strongly limited in his movement (see also Fig. 5). The isotopic substitution, shown in Fig. 4, in which the blue bars identify the $\mathrm{H}$ atom when substituted with its $\mathrm{D}$ isotope, confirms the attribution to $\mathrm{H}$ of the mode at $364 \mathrm{~cm}^{-1}$.

In the same figure, it is possible to identify the modes to which $\mathrm{N}$ and $\mathrm{B}$ give the most important contributions (gray and red bars, respectively). Also in this case, the difference between $\mathrm{CHB}$ and $\mathrm{CHN}$ is large: $\mathrm{N}$ contributes to many modes, of which only the three most important are indicated in the figure. (The peak at $615 \mathrm{~cm}^{-1}$ is actually a superposition of a couple of $E$ modes with a $A_{1}$ mode, differing by less than $1 \mathrm{~cm}^{-1}$.)

The $B$ modes in CHB are on the contrary easily identified at 1411,1348 and $364 \mathrm{~cm}^{-1}$ (see animations). It is worth of note that the low wavenumber B mode is strongly correlated with the $\mathrm{H}$ bending, as the animation also confirms.

Let us consider now how the relevant peaks depend on the defect concentration: the $\mathrm{C}-\mathrm{H}$ stretching of $\mathrm{CHB}$ shifts by $49 \mathrm{~cm}^{-1}$ (from 3562 to $3513 \mathrm{~cm}^{-1}$ ), the one of CHN by $17 \mathrm{~cm}^{-1}$ (from 3480 and $3497 \mathrm{~cm}^{-1}$ ); as regards the $\mathrm{C}-\mathrm{H}$ bending, they shift by 62 and $30 \mathrm{~cm}^{-1}$ for CHB (from 1804 to 1866 , and from 396 to $266 \mathrm{~cm}^{-1}$ ) and by only $4 \mathrm{~cm}^{-1}$ for CHN (1586 to $1590 \mathrm{~cm}^{-1}$ ) in going from $\mathrm{S}_{64}$ and $\mathrm{S}_{216}$. So shifts are much larger for $\mathrm{CHB}$ than for $\mathrm{CHN}$. The $\mathrm{H}$ position, that in CHN is strongly constrained by symmetry, is on the contrary in a general position for CHB. This general
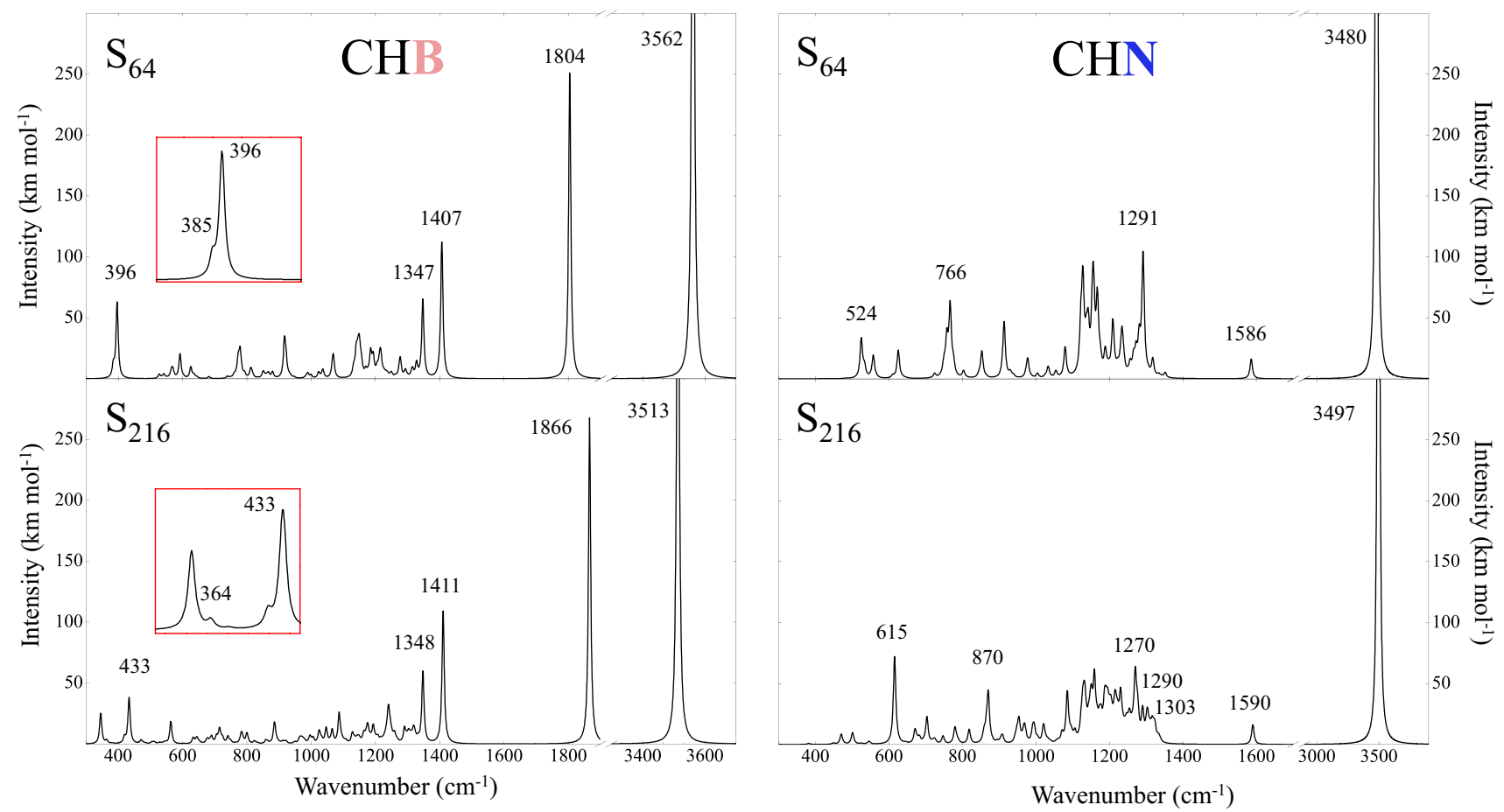

Fig. 3 B3LYP simulated IR spectra of the CHN (left panels) and CHB (right panels) defects in diamond. The top panels refer to $\mathrm{S}_{64}$, the bottom ones to $\mathrm{S}_{216}$ 

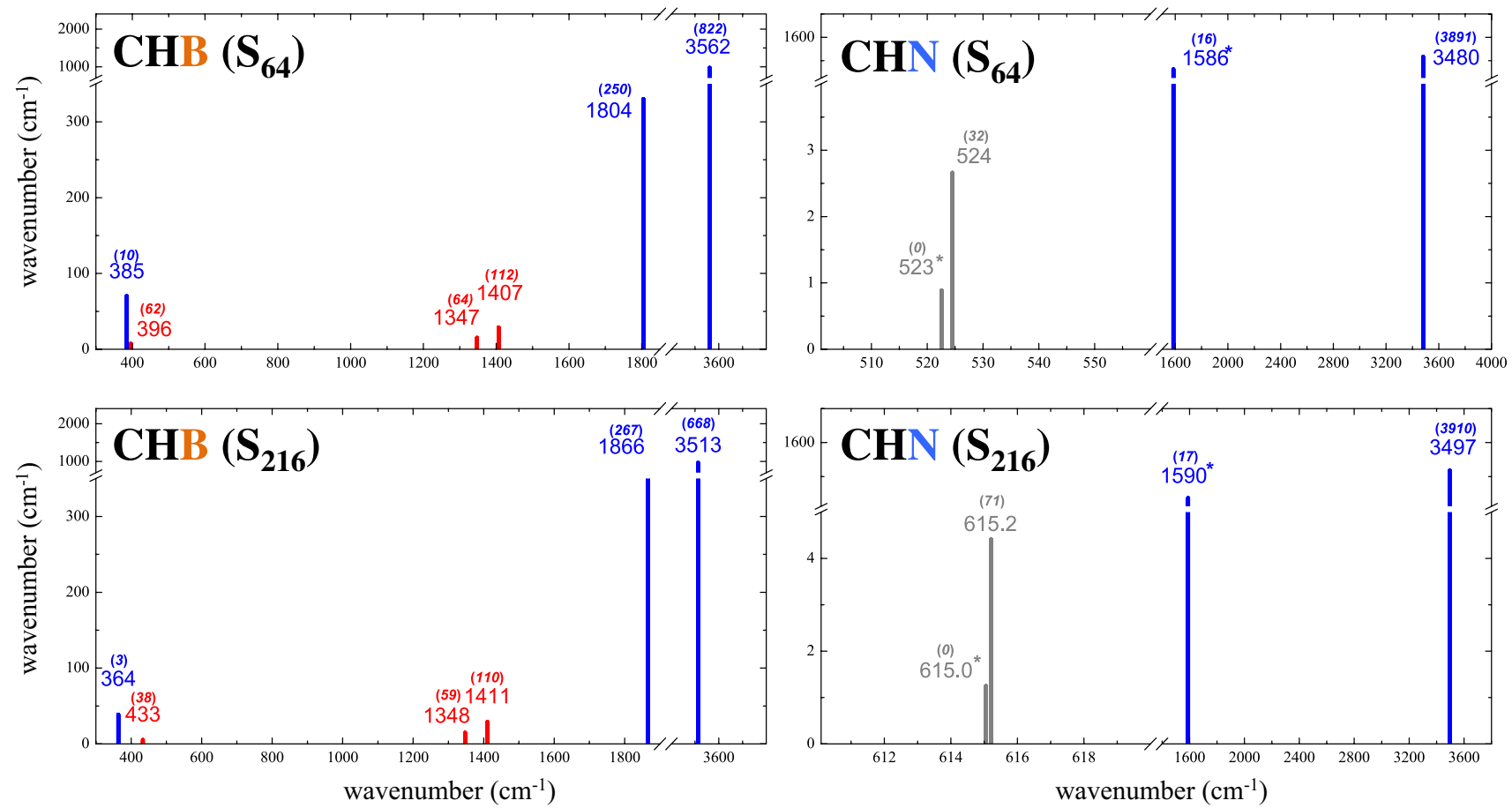

Fig. 4 Effect of the isotopic substitutions ${ }^{11} \mathrm{~B} \rightarrow{ }^{10} \mathrm{~B}$ (red bars), ${ }^{14} \mathrm{~N} \rightarrow$ ${ }^{15} \mathrm{~N}$ (gray bars) and $\mathrm{H} \rightarrow \mathrm{D}$ (blue bars) in the $\mathrm{S}_{64}$ (top panels) and $\mathrm{S}_{216}$ (bottom panels) supercells. The horizontal axis reports the frequen-

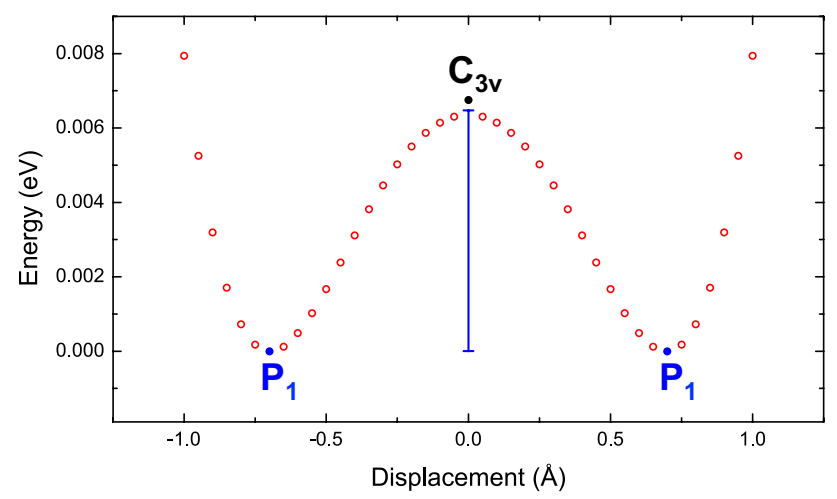

Fig. 5 When frequencies of CHB are computed keeping the $\mathrm{C}_{3 v}$ symmetry, a negative eigenvalue appears at $-374.35 \mathrm{~cm}^{-1}$. The figure shows the PES obtained following the corresponding eigenvector. The two $\mathrm{P}_{1}$ minimum points are $0.0065 \mathrm{eV}$ below the maximum. Data refer to $\mathrm{S}_{64}$

position turns out to strongly depend on the defect concentration, as discussed above. Note, however, that the two $B$ modes at 1411 and $1348 \mathrm{~cm}^{-1}$, which are not correlated with the $\mathrm{H}$ movement, essentially do not depend on the defect concentration.

As it is well known, the $\mathrm{CH}$ stretching is in general strongly anharmonic, and essentially independent from the other normal modes. Table 2, in the first four columns,

cies with the standard isotopes as in Fig. 3, the vertical axis the shift due to the isotopic substitution. The asterisks indicate doubly degenerate $\mathrm{E}$ levels. The IR intensities of the modes are reported in brackets

reports the results of the numerical solution of the onedimensional Schrodinger equation, as described in Sect. 2. In the same table, also the harmonic frequency $\omega_{H}$ obtained by diagonalizing the Hessian matrix, and using the one point formula (see Eq. 2) is reported. Let us consider first CHN. The fundamental anharmonic wavenumber is $3408 \mathrm{~cm}^{-1}$, about $64 \mathrm{~cm}^{-1}$ lower than the harmonic frequency $\left(\omega_{e}\right.$, at $3471 \mathrm{~cm}^{-1}$, see Eqs. 4 and 5). This number is $8 \mathrm{~cm}^{-1}$ lower than $\omega_{H}^{1}$, the frequency obtained by diagonalizing the full hessian matrix with the two-points formula (see Eq. 7; $\omega_{e}$, on the contrary, is obtained by computing 7 points on the one-dimensional PES). The relatively good agreement confirms that in this case: (1) the movement is along the onedimensional C-H coordinate; (2) that the PES is not very anharmonic.

The situation is quite different for CHB: here the difference between $\omega_{e}$ and $\omega_{H}^{1}$, is larger than $100 \mathrm{~cm}^{-1}$, showing that in this case the hypotheses (1) and (2) are no more valid, and the simple estimate of the anharmonicity along the $\mathrm{C}-\mathrm{H}$ bond is not very reliable.

Going back to the anharmonic $\mathrm{CH}$ stretching of $\mathrm{CHN}$, Table 2 shows that it is $260 \mathrm{~cm}^{-1}$ higher than the one in $\mathrm{VN}_{3} \mathrm{H}$, and also higher than many of the ones characterizing the families of $\mathrm{VH}_{x}$ and $\mathrm{VN}_{x} \mathrm{H}_{y}$ defects, investigated by some of the present authors in previous papers [22, 24], as in these cases the opposite wall toward which is moving $\mathrm{H}$ 
Table $2 \mathrm{C}-\mathrm{H}$ bond length $\left(\mathrm{R}_{\mathrm{CH}}\right.$, in $\AA$ ), fundamental anharmonic and first overtone $\left(\omega_{e 1}\right.$ and $\left.\omega_{e 2}\right)$, anharmonic constant $\left(\omega_{e} \chi_{e}\right)$ and the fundamental harmonic frequency $\left(\omega_{e}\right)$ for $\mathrm{CHB}$ and $\mathrm{CHN}$ obtained by using the $\mathrm{S}_{64}$ supercells and the B3LYP functional. The $\omega_{H}^{1}$ values are the harmonic frequencies obtained by diagonalizing the full Hes- sian matrix, built by using the two points formula (see Eqs. 1 and 2, respectively). For comparison, the $\mathrm{CH}$ stretching of the $\mathrm{VN}_{3} \mathrm{H}$ defect (see Ref. [7], in which the same basis set and functional have been used) is reported. Wavenumbers in $\mathrm{cm}^{-1}$

\begin{tabular}{lllllll}
\hline & $\mathrm{R}_{\mathrm{CH}}$ & $\omega_{e 1}$ & $\omega_{e 2}$ & $\omega_{e} \chi_{e}$ & $\omega_{e}$ & $\omega_{H}^{1}$ \\
\hline $\mathrm{CHB}($ here $)$ & 1.066 & 3394 & 6729 & 30 & 3455 & 3562 \\
$\mathrm{CHN}($ here $)$ & 1.038 & 3408 & 6751 & 32 & 3471 & 3480 \\
$\mathrm{CHN}\left(\mathrm{VN}_{3} \mathrm{H}\right)$ & 1.074 & 3132 & 6127 & 69 & 3271 \\
\hline
\end{tabular}
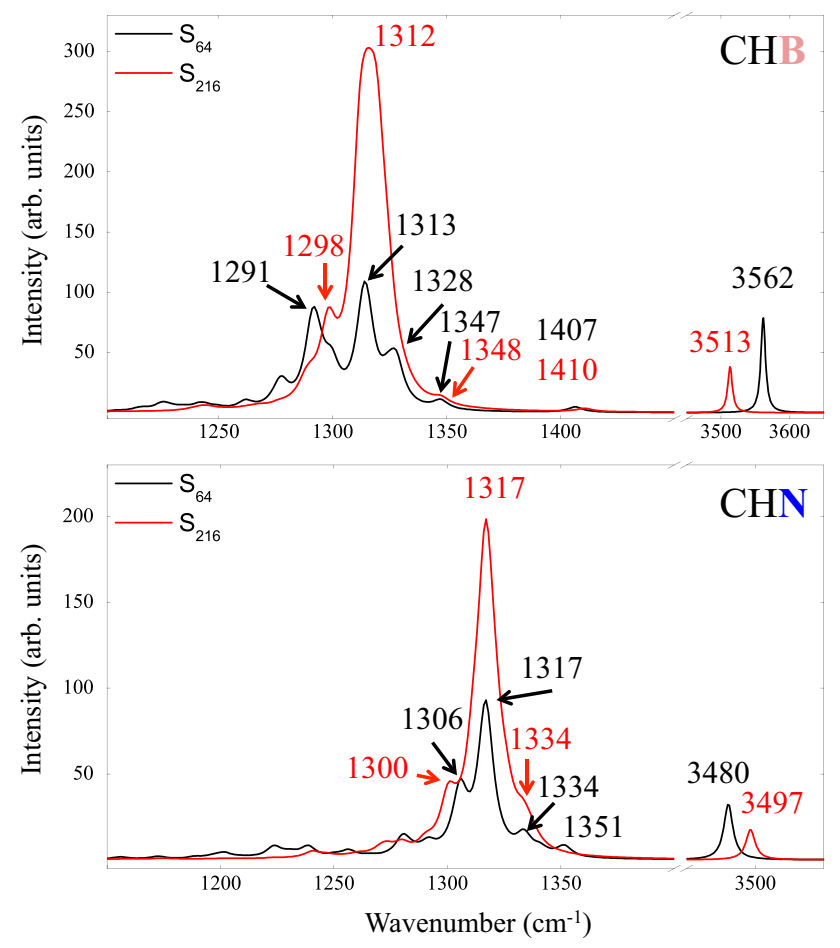

Fig. 6 The Raman spectra of CHB (top) and CHN (bottom) obtained with $\mathrm{S}_{64}$ (black lines) and $\mathrm{S}_{216}$ (red lines). Both intensities are fitted with a Lorentzian function with a full width at half maximum of $8 \mathrm{~cm}^{-1}$

are farther away, on the opposite side of the vacancy. This distance should make the region of the $\mathrm{CHN}$ stretching less crowded, and then the stretching itself more easy to identify (see conclusions).

\subsubsection{Raman spectrum}

The Raman spectra of $\mathrm{CHN}$ and CHB, obtained at two defect concentrations, are shown in Fig. 6. The position of the peaks is obviously the same as in the IR spectrum. The relative intensity is on the contrary very different and can be used as an additional source of information and identification for these defects. Unfortunately, we have been unable to find any experimental Raman spectrum in the literature so that we propose the present ones for future comparison.

\subsection{Comparison with previous calculations}

Our calculated fundamental harmonic stretching of $\mathrm{CHN}$ is $3471 \mathrm{~cm}^{-1}$, which red shifts to $3408 \mathrm{~cm}^{-1}$ when anharmonicity is taken into account.

Goss et al. have estimated [9] it at around $3309 \mathrm{~cm}^{-1}$. Our bending mode is around $1866 \mathrm{~cm}^{-1}$, while they obtained $1404 \mathrm{~cm}^{-1}$.

The same authors have calculated the stretching and wagging frequencies of the CHB defect at 2664 and $1975 \mathrm{~cm}^{-1}$, respectively, while our computed ones are at 3394 and 1410 $\mathrm{cm}^{-1}$.

Frequencies for the CHB [6] and the CHN [9] centers were obtained by Goss and collaborators by using their own AIMPRO code [58] with both cluster and supercell approaches, using LDA, a Gaussian-type basis set and pseudopotentials. The extremely large difference between the present and Goss results cannot be attributed only to the use of LDA, which is, however, well known to underestimate vibrational frequencies, especially in strongly $\mathrm{H}$-bonded systems [59].

\section{Conclusion}

The structural, electronic, energetic and vibrational features of the $\mathrm{CHN}$ and $\mathrm{CHB}$ point defects in diamond have been estimated through quantum-mechanical calculations. Both defects are closed shell in the ground state configuration. According to our calculations, in the CHN center hydrogen points directly toward the lone pair of the substitutional nitrogen atom, leading to a $\mathrm{C}_{3 v}$ symmetry. In $\mathrm{CHB}$, on the contrary, $\mathrm{H}$ falls off from the axis between boron and carbon, leading to a P1 symmetry of the defect, as a consequence of the strong repulsion between $\mathrm{B}$ and $\mathrm{H}$, both positively charged.

IR and Raman spectra of both defects have been simulated, with a particular attention to the $\mathrm{C}-\mathrm{H}$ stretching and bending modes that might be used as fingerprints of these 
defects. Frequency shifts upon isotopic substitutions have been calculated, as well as graphical animation of the normal modes [56, 57].

The $\mathrm{C}-\mathrm{H}$ stretching frequency of $\mathrm{CHN}$ red shifts by $63 \mathrm{~cm}^{-1}$ to $3408 \mathrm{~cm}^{-1}$, when anharmonicity is taken into account through a one-mode correction.

The experimental peak [3] at $3394 \mathrm{~cm}^{-1}$, peculiar of $\mathrm{Ib}$ diamonds (in which nitrogen is mainly single substitutional), is very close to our $\mathrm{C}-\mathrm{H}$ stretching mode of the $\mathrm{CHN}$ center, the difference being only $14 \mathrm{~cm}^{-1}$. So one possible attribution of this experimental peak is the $\mathrm{CHN}$ defect.

For the CHB center, the situation is not as clear as for the $\mathrm{CHN}$ defect, since no experimental data match our calculated frequencies. In a previous experimental work [3], it has been reported that IIb diamonds (rich in boron) do not show any significant IR peak in the region of interest. Note, however, from Fig. 3 that the $\mathrm{CH}$ stretching in $\mathrm{CHB}$ is very intense, and should be clearly visible in experiments.

Acknowledgements RD and FSG acknowledge the CINECA award (HP10CTG8YY) under the ISCRA initiative, for the availability of highperformance computing resources and support.

\section{References}

1. Jackman RB (2003) Special issue: diamond electronics-preface. Semicond Sci Technol 18(3):1-2

2. Nemanich RJ, Carlisle JA, Hirata A, Haenen K (2014) CVD diamond-research, applications, and challenges. MRS Bull 39(06):490-494

3. Woods GS, Collins AT (1983) Infrared absorption spectra of hydrogen complexes in type I diamonds. J Phys Chem Solids 44(5):471-475

4. Hainschwang T, Notari F, Fritsch E, Massi L (2006) Natural, untreated diamonds showing the $\mathrm{A}, \mathrm{B}$ and $\mathrm{C}$ infrared absorptions ("ABC Diamonds"), and the $\mathrm{H}_{2}$ absorption. Diam Relat Mater 15(10):1555-1564

5. Kaiser W, Bond WL (1959) Nitrogen, a major impurity in common type I diamond. Phys Rev 115(4):857

6. Goss JP, Jones R, Heggie MI, Ewels CP, Briddon PR, Öberg S (2002) Theory of hydrogen in diamond. Phys Rev B 65(11): 115207

7. Gentile F, Salustro S, Causá M, Erba A, Carbonniére P, Dovesi $\mathrm{R}$ (2017) The $\mathrm{VN}_{3} \mathrm{H}$ defect in diamond. A quantum mechanical investigation of the structural, electronic and vibrational properties. Phys Chem Chem Phys 1(4):1-2

8. Goss JP, Briddon PR, Hill V, Jones R, Rayson MJ (2014) Identification of the structure of the $3107 \mathrm{~cm}^{-1} \mathrm{H}$-related defect in diamond. J Phys Condens Matter 26:145801

9. Goss Jonathan P, Briddon Patrick R (2011) Calculated strain response of vibrational modes for $\mathrm{H}$-containing point defects in diamond. Phys Chem Chem Phys 13(24):11488-11494

10. Dovesi R, Erba A, Orlando R, Zicovich-Wilson CM, Civalleri B, Maschio L, Rérat M, Casassa S, Baima J, Salustro S, Kirtman B (2018) Quantum-mechanical condensed matter simulations with CRYSTAL. WIREs Comput Mol Sci 8. https://doi.org/10.1002/ wcms. 1360

11. Maschio L, Kirtman B, Orlando R, Rérat M (2012) Ab initio analytical infrared intensities for periodic systems through a coupled perturbed Hartree-Fock/Kohn-Sham method. J Chem Phys 137(20):204113

12. Maschio L, Kirtman B, Rérat M, Orlando R, Dovesi R (2013) Ab Initio analytical Raman intensities for periodic systems through a coupled perturbed Hartree-Fock/Kohn-Sham method in an atomic orbital basis. II. Validation and comparison with experiments. J Chem Phys 139(16):164102

13. Carteret C, De La Pierre M, Dossot M, Pascale F, Erba A, Dovesi $\mathrm{R}$ (2013) The vibrational spectrum of $\mathrm{CaCO}_{3}$ aragonite: a combined experimental and quantum-mechanical investigation. J Chem Phys 138(1):014201

14. Maschio L, Kirtman B, Salustro S, Zicovich-Wilson CM, Orlando R, Dovesi R (2013) Raman spectrum of pyrope garnet. A quantum mechanical simulation of frequencies, intensities, and isotope shifts. J Phys Chem A 117(45):11464-11471

15. Prencipe M, Maschio L, Kirtman B, Salustro S, Erba A, Dovesi $\mathrm{R}$ (2014) Raman spectrum of $\mathrm{NaAlSi}_{2} \mathrm{O}_{6}$ jadeite. A quantum mechanical simulation. J Raman Spectrosc 45:703-709

16. Baima J, Zelferino A, Olivero P, Erba A, Dovesi R (2016) Raman spectroscopic features of the neutral vacancy in diamond from $\mathrm{Ab}$ initio quantum-mechanical calculations. Phys Chem Chem Phys 18(3):1961-1968

17. Zelferino A, Salustro S, Baima J, Lacivita V, Orlando R, Dovesi R (2016) The electronic states of the neutral vacancy in diamond: a quantum mechanical approach. Theor Chem Acc 135(3):1-11

18. Salustro S, Erba A, Zicovich-Wilson CM, Nöel Y, Maschio L, Dovesi R (2016) Infrared and Raman spectroscopic features of the self-interstitial defect in diamond from exact-exchange hybrid DFT calculations. Phys Chem Chem Phys 120:21288-21295

19. Salustro S, Ferrari AM, Gentile FS, Desmarais JK, Rérat M, Dovesi R (2018) Characterization of the B-center defect in diamond through the vibrational spectrum: a quantum mechanical approach. J Phys Chem A 122(2):594-600

20. Salustro S, Ferrari AM, Orlando R, Dovesi R (2017) Comparison between cluster and supercell approaches: the case of defects in diamond. Theor Chem Acc 136:42

21. Salustro S, Gentile FS, D’Arco P, Civalleri B, Rérat M, Dovesi R (2018) Hydrogen atoms in the diamond vacancy defect. A quantum mechanical vibrational analysis. Carbon 129:349-356

22. Salustro S, Gentile F, Erba A, Carbonniére P, El-Kelany K, Dovesi $\mathrm{R}$ (2018) The characterization of the $\mathrm{VN}_{x} \mathrm{H}_{y}$ defects in diamond through the infrared vibrational spectrum. A quantum mechanical investigation. Carbon 132:210-219

23. Ferrari A M, Salustro S, Gentile F, Mackrodt W, Dovesi R (2018) Substitutional nitrogen atom in diamond. A quantum mechanical investigation of the electronic and spectroscopic properties. Carbon 134:354-365

24. Gentile F, Salustro S, Desmarais J, Ferrari A M, D'Arco P, Dovesi R (2018) Vibrational spectroscopy of hydrogens in diamond: a quantum mechanical treatment. Phys Chem Chem Phys 20(17): 11930-11940

25. Becke A D (1993) Density-functional thermochemistry. III. The role of exact exchange. J Chem Phys 98(7):5648-5652

26. Lee C, Yang W, Parr R (1988) Development of the Colle-Salvetti correlation-energy formula into a functional of the electron density. Phys Rev B 37(2):785-789

27. Dovesi R, Orlando R, Erba A, Zicovich-Wilson C M, Civalleri B, Casassa S, Maschio L, Ferrabone M, De La Pierre M, D’Arco P, Noël Y, Causà M, Rérat M, Kirtman B (2014) CRYSTAL14: a program for the $A b$ Initio investigation of crystalline solids. Int $\mathbf{J}$ Quantum Chem 114:1287

28. Hehre WJ, Ditchfield R, Pople JA (1972) Self-consistent molecular orbital methods. XII. Further extensions of gaussian type basis sets for use in molecular orbital studies of organic molecules. J Chem Phys 56(5):2257-2261 
29. Francl MM, Petro WJ, Hehre WJ, Binkley JS, Gordon MS, DeFrees DJ, Pople JA (1982) Self-consistent molecular orbital methods. XXIII. A polarization-type basis set for second-row elements. J Chem Phys 77(7):3654-3665

30. Dirac P (1930) A theory of electrons and protons. Proc R Soc A 126(801):360-365

31. Perdew JP, Burke K, Ernzerhof M (1996) Generalized gradient approximation made simple. Phys Rev Lett 77(18):3865-3868

32. Adamo C, Barone V (1998) Toward chemical accuracy in the computation of NMR shieldings: the PBE0 model. Chem Phys Lett 298(1):113-119

33. Krukau AV, Vydrov OA, Izmaylov AF, Scuseria GE (2006) Influence of the exchange screening parameter on the performance of screened hybrid functionals. J Chem Phys 125(22):224106-224106

34. Dovesi R, Saunders VR, Roetti C, Orlando R, Zicovich-Wilson CM, Pascale F, Civalleri B, Doll K, Harrison NM, Bush IJ, D'Arco P, Llunel M, Causà M, Noël Y, Maschio L, Erba A, Rérat M, Casassa S (2018) CRYSTAL17 user's manual. http://www. crystal.unito.it/Manuals/crystal17.pdf

35. Pascale F, Zicovich-Wilson CM, Lòpez Gejo F, Civalleri B, Orlando R, Dovesi R (2004) The calculation of the vibrational frequencies of the crystalline compounds and its implementation in the CRYSTAL code. J Comput Chem 25(6):888-897

36. Zicovich-Wilson CM, Pascale F, Roetti C, Saunders VR, Orlando $\mathrm{R}$, Dovesi R (2004) Calculation of the vibration frequencies of $\alpha$ -quartz: the effect of hamiltonian and basis set. J Comput Chem 25(15):1873-1881

37. Erba A, Ferrabone M, Orlando R, Dovesi R (2013) Accurate dynamical structure factors from Ab initio lattice dynamics: the case of crystalline silicon. J Comput Chem 34:346-354

38. Baima J, Ferrabone M, Orlando R, Erba A, Dovesi R (2016) Thermodynamics and phonon dispersion of pyrope and grossular silicate garnets from Ab Initio simulations. Phys Chem Miner 43:137-149

39. Pascale F, Zicovich-Wilson CM, Orlando R, Roetti C, Ugliengo P, Dovesi $\mathrm{R}$ (2005) Vibration frequencies of $\mathrm{Mg}_{3} \mathrm{Al}_{2} \mathrm{Si}_{3} \mathrm{O}_{12}$ pyrope. An Ab Initio study with the CRYSTAL code. J Phys Chem B 109(13):6146-6152

40. Orlando R, Torres FJ, Pascale F, Ugliengo P, Zicovich-Wilson $\mathrm{CM}$, Dovesi R (2006) Vibrational spectrum of katoite $\mathrm{Ca}_{3} \mathrm{Al}_{2}$ $\left[(\mathrm{OH})_{4}\right]_{3}$ : a periodic Ab initio study. J Phys Chem B 110:692-701

41. Pascale F, Tosoni S, Zicovich-Wilson CM, Ugliengo P, Orlando $\mathrm{R}$, Dovesi R (2004) Vibrational spectrum of brucite $\mathrm{Mg}(\mathrm{OH})_{2}$ : a periodic $\mathrm{Ab}$ initio quantum mechanical calculation including $\mathrm{OH}$ anharmonicity. Chem Phys Lett 396:308-315

42. Demichelis R, Noël Y, Civalleri B, Roetti C, Ferrero M, Dovesi R (2007) The vibrational spectrum of $\alpha$-AlOOH diaspore: an Ab initio study with the crystal code. J Phys Chem B 111(31):9337-9346

43. Barrow GM (1962) Introduction to molecular spectroscopy. McGraw-Hill, New York

44. Hess BA, Schaad LJ, Carsky P, Zahradnik R (1986) Ab initio calculations of vibrational spectra and their use in the identification of unusual molecules. Chem Rev 86:709-730
45. Maschio L, Kirtman B, Rérat M, Orlando R, Dovesi R (2013) Comment on $A b$ Initio analytical infrared intensities for periodic systems through a coupled perturbed Hartree-Fock/KohnSham method [J Chem Phys 137, 204113 (2012)]. J Chem Phys 139:167101

46. Maschio L, Kirtman B, Rérat M, Orlando R, Dovesi R (2013) $\mathrm{Ab}$ initio analytical raman intensities for periodic systems through a coupled perturbed Hartree-Fock/Kohn-Sham method in an atomic orbital basis. I. Theory. J Chem Phys 139:164101

47. Maschio L, Kirtman B, Rérat M, Orlando R, Dovesi R (2013) $\mathrm{Ab}$ initio analytical Raman intensities for periodic systems through a coupled perturbed Hartree-Fock/Kohn-Sham method in an atomic orbital basis. II. Validation and comparison with experiments. J Chem Phys 139:164102

48. Ferrero M, Rérat M, Orlando R, Dovesi R (2008) The calculation of static polarizabilities of periodic compounds. The implementation in the CRYSTAL code for 1D, 2D and 3D systems. J Comput Chem 29:1450-1459

49. Ferrero M, Rérat M, Orlando R, Dovesi R (2008) Coupled perturbed Hartree-Fock for periodic systems: the role of symmetry and related computational aspects. J Chem Phys 128:014110

50. McQuarrie DA, Simon JD (1977) Physical chemistry: a molecular approach. University Science Books, Sausalito

51. Duncan JL, McKean DC, Tortoand I, Brown A, Ferguson A M (1988) Infrared studies of $\mathrm{CH}$ and CD stretching anharmonicity. J Chem Soc Faraday Trans 2 84(9):1423-1442

52. Myrick ML, Greer AE, Nieuwland AA, Priore RJ, Scaffidi J, Andreatta D, Colavita P (2008) Birge-Sponer estimation of the $\mathrm{C}-\mathrm{H}$ bond dissociation energy in chloroform using infrared, nearinfrared, and visible absorption spectroscopy. An experiment in physical chemistry. J Chem Educ 85(9):1276

53. Cardona M, Thewalt MLW (2005) Isotope effects on the optical spectra of semiconductors. Rev Mod Phys 77(4):1173

54. Bjørheim TS, Arrigoni M, Gryaznov D, Kotomin E, Maier Joachim (2015) Thermodynamic properties of neutral and charged oxygen vacancies in $\mathrm{BaZrO}_{3}$ based on first principles phonon calculations. Phys Chem Chem Phys 17(32):20765-20774

55. Bjørheim TS, Arrigoni M, Saeed SW, Kotomin E, Maier Joachim (2016) Surface segregation entropy of protons and oxygen vacancies in $\mathrm{BaZrO}_{3}$. Chem Mater 28(5):1363-1368

56. Animations of the vibrational modes of the $\mathrm{CHN}$ defect in diamond can be found at the following link. http://www.pmmp. jussieu.fr/yves/variousdefects/animations/?name=04_NHC. $\mathrm{xyz} \& \mathrm{spt}=04 \_$NHC.spt

57. Animations of the vibrational modes of the CHB defect in diamond can be found at the following link. http://www.pmmp. jussieu.fr/yves/variousdefects/animations/?name $=05$ _BHC. xyz\&spt $=05 \_B H C . s p t$

58. Briddon PR, Jones R (2000) LDA calculations using a basis of Gaussian orbitals. Phys Status Solidi B 217(1):131-171

59. Ugliengo P, Pascale F, Merawa M, Labéguerie P, Tosoni S, Dovesi $R$ (2004) Infrared spectra of hydrogen-bonded ionic crystals: $\mathrm{Ab}$ initio study of $\mathrm{Mg}(\mathrm{OH})_{2}$ and $\beta-\mathrm{Be}(\mathrm{OH})_{2}$. J Phys Chem B 108(36):13632-13637 


\section{Hydrogen, Boron and Nitrogen Atoms in Diamond. A Quantum Mechanical Vibrational Analysis Supplemental Material}

Francesco Silvio Gentile, ${ }^{1}$ Simone Salustro, ${ }^{1}$ Giulio Di Palma, ${ }^{1}$ Mauro Causà, ${ }^{2}$ Philippe D'Arco, ${ }^{3}$ and Roberto Dovesi ${ }^{1}$

${ }^{1}$ Dipartimento di Chimica, Università di Torino and NIS (Nanostructured Interfaces and Surfaces) Centre, Via P. Giuria 5, 10125 Torino, Italy

${ }^{2}$ Dipartimento di Ingengeria Chimica, dei Materiali e delle Produzioni Industriali DICMAPI,

Università degli Studi di Napoli Federico II, Piazzale Vincenzo Tecchio 80, 80125, Napoli, Italy

${ }^{3}$ Institut des Sciences de la Terre de Paris (UMR 7193 UPMC-CNRS), UPMC, Sorbonne Université, Paris, France

I. $\triangle$ G OF HYDROGENATION REACTIONS IN C $\Sigma$ DIAMOND DEFECTS

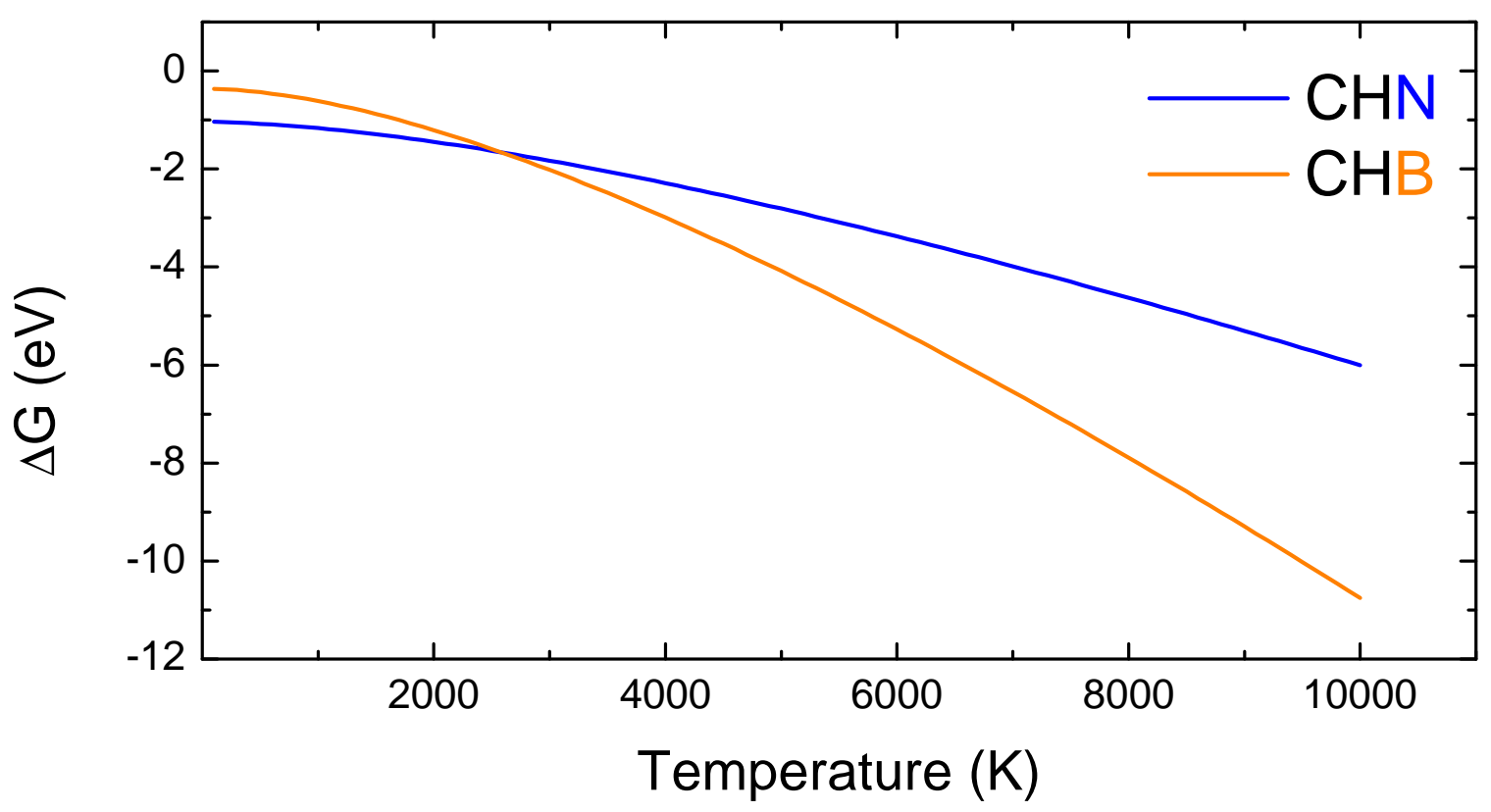

Figure S1: The hydrogenation free energy $\Delta \mathrm{G}$ of $\mathrm{CB}$ and $\mathrm{CN}$ defects in diamond. The explored temperature range is 100 - $10000 \mathrm{~K}$. Data refer to $\mathrm{S}_{64}$ supercell.

The hydrogenation free energy $\Delta \mathrm{G}$ (see equation 9 in the manuscript, where $\mathrm{C} \Sigma$ defects react with $\mathrm{H}$ radical), are derived from $\Gamma$-point only phonon calculations. $\Delta \mathrm{G}$ is evaluated in the wide interval from 100 to $10000 \mathrm{~K}$. All energies are in eV for a comparison with the hydrogenation energies $\Delta \mathrm{E}$ reported in Table $\mathrm{I}$ of the manuscript. $\Delta \mathrm{G}$ are calculated according to Equation $\mathrm{S} 1$, where $\mathrm{E}_{e l}$ is the electronic energy, $\mathrm{E}_{0}$ is the zero point energy, $\mathrm{E}_{T}$ is the translational contribute and PV and TS are the enthalpic and entropic contributions. In the isolated $\mathrm{H}$ atom $\mathrm{E}_{0}, \mathrm{E}_{T}$ and -TS are null, and the PV term (for one mole of ideal atom gas) is equal to RT.

$$
G=E_{e l}+E_{0}+E_{T}+P V-T S,
$$

$\Delta \mathrm{G}$ is negative in all cases, confirming the spontaneous character of the reactions. At low temperature the CHN curve is lower than the $\mathrm{CHB}$ one. At about $2600 \mathrm{~K}$ the two curves cross. Thereafter the CHB curve is lower than CHN, due to a larger slope of the curve, indicating an higher stability of the hydrogenation reaction in extreme conditions, tipically at the high temperatures 
observed in the mantle, where these defects might form. 